\title{
Inference for Progressive Type-II Censoring \\ Statistical Inference for the Exponentiated General Class \\ Based on Progressive Type-II Censoring
}

Gannat R. AL-Dayian ${ }^{\text {a }}$ Lamya A. Baharith ${ }^{\text {; }}$ Hadeel S. Klakattawi ${ }^{\text {c }}$

${ }^{a}$ Department of Statistics, Faculty of Commerce, AL-Azhar University, Cairo, Egypt

P.O.Box $1152810^{\text {th }}$ Av. Naser City, Cairo. E-mail: galdayian@hotmail.com

${ }^{\mathrm{b}}$ Department of Statistics, Faculty of Science, King Abdulaziz University, Jeddah, Saudi Arabia

P.O.Box 42805 Jeddah 21551 E-mail: Ibaharith@kau.edu.sa

${ }^{\text {c} D e p a r t m e n t ~ o f ~ S t a t i s t i c s, ~ F a c u l t y ~ o f ~ S c i e n c e, ~ K i n g ~ A b d u l a z i z ~ U n i v e r s i t y, ~ J e d d a h, ~}$ Saudi Arabia

P.O.Box 42805 Jeddah 21551. E-mail: hklakattawi@kau.edu.sa 


\title{
Statistical Inference for the Exponentiated General Class Based on Progressive Type-II Censoring
}

\author{
Gannat R. AL-Dayian*, Lamya A. Baharith**and Hadeel S. Klakattawi** \\ *Department of Statistics, Faculty of Commerce, AL-Azhar University, Cairo, Egypt \\ **Department of Statistics, Faculty of Science, King Abdulaziz University, Jeddah, Saudi Arabia
}

\begin{abstract}
An exponentiated general class (EGC) of distributions was considered as the underlying population of this study. Based on a progressive Type-II censored sample, the maximum likelihood and Bayesian estimation of the parameters, the reliability and hazard rate functions of an EGC were studied. Bayesian estimations were conducted under the squared error and linear-exponential (LINEX) loss functions. The maximum likelihood and Bayes predictors of the life lengths of all censored units in the progressive sample are discussed. In addition, the Bayes predictors of future observables were obtained based on the two-sample prediction. A numerical example is given to illustrate some of the results.
\end{abstract}

Keywords Exponentiated general class; Progressive Type-II censored sample; squared error and LINEX loss functions; Maximum likelihood estimation (prediction); Bayesian estimation (prediction); Two-sample prediction.

\section{Introduction}

Currently, life testing studies are becoming more important in many fields to obtain information on the time of occurrence for an event of interest. Many distributions are used as lifetime models. Adding one or more parameters to a distribution function makes the resulting distribution more flexible for modeling the data. That is, adding a parameter to a cumulative lifetime distribution function $G(t ; \underline{\eta})$ by exponentiation produces distributions that have a cumulative distribution function $(\mathrm{CDF})$ of the form $F(t ; \theta, \underline{\eta})=[G(t ; \underline{\eta})]^{\theta}$. Such a distribution is 
called an exponentiated model, and if $\theta$ is a positive, this model is also referred to in the literature as Lehman alternatives, as has been recognized by Gupta et al. (1998).

It is interesting to examine families of lifetime distributions with useful properties that enable them to describe lifetimes of units. This study aimed to discuss a generalized family of distributions, called the exponentiated general class (EGC). The EGC was suggested based on the general class that was used by AL-Hussaini (1999) and Soliman (2002) as a lifetime model.

This class has been modified by adding a parameter by exponentiation to its CDF, which is regarded as an added advantage over the general class. It should be mentioned that the EGC was proposed by Abdel-Hamid and AL-Hussaini (2009) as the lifetime of a product in a stepstress model and was considered as an exponentiated distribution. The EGC generalizes many distributions that are commonly used in life testing and in the analysis of lifetime data. Such distributions are considered by many authors as 'generalized' distributions. The word exponentiated instead of generalized may lend more clarification, expressiveness and accuracy. Distributions that can be derived from the EGC include the following: exponentiated exponential (EE), exponentiated Weibull (EW), Burr X (exponentiated Rayleigh), exponentiated modified Weibull, exponentiated Pareto (EP), exponentiated gamma, among others. That is, the results obtained for the EGC could be applied to those lifetime distributions.. In many studies, complete information on the lifetimes of all of the experimental units may not be available for several reasons.

The progressive Type-II censored sampling is an important and flexible scheme in lifetesting experiments in which the experimenter can remove some units at various stages during the experiments. This scheme is useful for saving time, effort and cost. 
Estimating the unknown parameters of the distributions is one of the important problems in statistical studies. In this paper, the maximum likelihood (ML) and Bayesian estimations are studied.

In addition, the general problem of statistical prediction (inferring the values of unknown observations or their functions based on the informative samples) has its uses in a variety of areas. The predictors of times to failures of units censored in multiple stages in a progressive censored sample are discussed in this paper. Raqab et al. (2010) studied this type of prediction. Moreover, Basak and Balakrishnan (2009) considered different predictors based on the progressive Type-II censored samples from the Pareto and the normal distributions. Furthermore, Madi and Raqab (2009) studied the Bayesian prediction based on a progressive sample from an EE distribution.

This paper is concentrated on Bayesian prediction bounds for future order statistics from an EGC using a two-sample scheme based on a progressive Type-II informative sample. Some related works can be found in Wu et al. (2006), Ali Mousa and Jaheen (2002) and Jaheen (2003). The focus of this paper was to estimate the parameters, reliability function (RF) and hazard rate function (HRF) as well as to predict two different situations based on the progressive Type-II censored scheme. The estimation and prediction were obtained for two cases:

(i) The EGC depends on the only unknown exponent parameter.

(ii) The EGC depends on a vector of unknown parameters.

The rest of the paper is organized as follows. In Section 2, the EGC and the related model are discussed. ML estimations are presented in Section 3. In Section 4, Bayesian estimations under the squared error (SE) and Linear-exponential (LINEX) loss functions are obtained. The ML and Bayes predictions of the lifetime lengths of all censored units in the progressive sample 
are discussed in Section 5. In Section 6, the Bayes predictors of future observables based on a two-sample scheme in which the informative sample is progressive Type-II censored and the future sample is order statistics is studied. When only the exponent parameter is unknown and in the case of an unknown vector of parameters, two cases are considered in each section. Finally, a numerical example is given in Section 7 to illustrate the method of estimation and the Bayes predictions of the lifetime lengths of all of the censored units in the progressive sample.

\section{The Exponentiated General Class}

If $G(t ; \underline{\eta})$ is a base CDF, then by adding one more parameter (say $\theta$ ) by exponentiation, the CDF of the exponentiated distribution $F(t ; \theta, \eta)$ takes one of the following forms:

(a) $F(t ; \theta, \underline{\eta})=[G(t ; \underline{\eta})]^{\theta}$, or

(b) $F(t ; \theta, \underline{\eta})=1-[1-G(t ; \underline{\eta})]^{\theta}$,

where $G(t ; \eta)$ is any CDF, and $\theta>0$ (see Kakade et al. (2008) and Persson and Ryden (2010)). It is worth noting that, in some studies, the exponentiated model in the form (1) is also known as a proportional reversed hazard model. However, the exponentiated model form in (2) is known as a proportional hazard model.

Any CDF for a random variable $T$ can be written in the following form:

$$
G(t)=1-\exp [-\lambda(t)]
$$

The probability density function (PDF) corresponding to (3) is

$$
g(t)=\lambda^{\prime}(t) \exp [-\lambda(t)]
$$

where $\lambda(t) \equiv \lambda(t ; \beta)=-\ln [1-G(t)]$, 
$\beta \in \Omega$ is a vector of parameters, and $\lambda(t)$ is assumed to be a continuous, monotone increasing, differentiable function such that $\lambda(t) \rightarrow 0$ as $t \rightarrow-\infty$ and $\lambda(t) \rightarrow \infty$ as $t \rightarrow \infty$ (see AbdelHamid and AL-Hussaini (2009) and AL-Hussaini and Al-Awadhi (2010)).

The EGC could be a special case of form (1) of the exponentiated model with the base CDF as the general class in (3). Suppose that $T$ is a positive random variable denoting the life length of a component having a CDF in (1) and a PDF as follows:

$f(t ; \theta, \underline{\eta})=\theta G^{\prime}(t ; \underline{\eta})[G(t ; \underline{\eta})]^{\theta-1}, t>0, \theta>0$

such that $G(t ; \underline{\eta})$ is a base CDF that depends only on $\underline{\eta}$, and $G(t ; \underline{\eta})$ is assumed to be an absolutely continuous CDF that is defined on the positive half of the real line as follows.

$G(t ; \underline{\eta})=1-\exp (-\lambda(t ; \underline{\eta})) \quad$ and $\quad G^{\prime}(t ; \underline{\eta})=\lambda^{\prime}(t ; \underline{\eta}) \exp (-\lambda(t ; \underline{\eta}))$

where $\lambda(t ; \underline{\eta})=-\ln \left\{1-[F(t ; \theta, \underline{\eta})]^{1}\right\}$

$(\theta, \underline{\eta}) \in \Omega, \theta>0, \underline{\eta}=\left(\eta_{1}, \eta_{2}, \ldots, \eta_{k}\right)$, where $\eta_{j}>0 ; j=1,2, \ldots, k, \Omega$ is a parameter space and $\lambda(t ; \eta)$ is a non-negative, continuous, monotone increasing and differential function of $t$ such that $\lambda(t ; \underline{\eta}) \rightarrow 0$ as $t \rightarrow 0^{+}$and $\lambda(t ; \underline{\eta}) \rightarrow \infty$ as $t \rightarrow \infty$.

The corresponding RF and HRF are given by the following, respectively:

$$
\begin{aligned}
& R(t ; \theta, \underline{\eta})=1-[G(t ; \underline{\eta})]^{\theta} \text { and } \\
& h(t ; \theta, \underline{\eta})=\frac{\theta \lambda^{\prime}(t ; \underline{\eta}) \exp (-\lambda(t ; \underline{\eta}))[G(t ; \underline{\eta})]^{\theta-1}}{1-[G(t ; \underline{\eta})]^{\theta}} .
\end{aligned}
$$

\section{Maximum Likelihood Estimation}

In this section, the parameters $\theta, \underline{\eta}, \mathrm{RF}$ and HRF at time $t_{0}$ are estimated based on the progressive Type-II censored sample from the EGC by using ML methods for two cases; in the 
first case, $\theta$ is the only unknown parameter, and in the second case all parameters, $\theta$ and $\underline{\eta}$ are unknown.

\subsection{Case (1): $\theta$ is unknown and $\underline{\eta}$ is known}

Assuming that $G(t)$ in (6) depends on known parameters, and then $F(t ; \theta)$ depends only on the unknown exponent parameter $\theta$.

Let $\underline{t}=\left(t_{1}, t_{2}, \ldots, t_{m}\right)$, with $t_{1} \leq t_{2} \leq \ldots \leq t_{m}$ denoting a progressive Type-II censored sample from $\operatorname{EGC}(\theta)$ with $R_{1}, R_{2}, \ldots, R_{m}$ being the progressive censoring scheme.

The joint PDF of all $m$ progressive Type-II censored order statistics $\underline{t}=\left(t_{1}, t_{2}, \ldots, t_{m}\right)$ is the following:

$$
L(\Omega ; \underline{t}) \propto \prod_{i=1}^{m} f\left(t_{i} ; \Omega\right)\left[1-F\left(t_{i} ; \Omega\right)\right]^{R_{i}}
$$

where $\Omega$ is a vector of parameters (see Balakrishnan and Aggarwala (2000)).

By substituting $F(t ; \theta, \underline{\eta}) \equiv F(t ; \theta)$ and $f(t ; \theta, \underline{\eta}) \equiv f(t ; \theta)$, which are given by (1) and (6), respectively, with known $\underline{\eta}$, the likelihood function (LF) based on the progressive Type-II censored sample from the EGC can be written as:

$L(\Omega ; \underline{t}) \propto \theta^{m} \prod_{i=1}^{m}\left[G\left(t_{i}\right)\right]^{\theta}\left\{1-\left[G\left(t_{i}\right)\right]^{\theta}\right\}^{R_{i}}$

where $G\left(t_{i}\right)$ and $G^{\prime}\left(t_{i}\right)$ are defined in (7) with known $\underline{\eta}$.

To derive the ML estimation of the unknown parameter $\theta$, denoted by $\hat{\theta}_{M L}$, we have to solve the first derivative of the logarithm (12) in $\theta$ as follows:

$\frac{m}{\theta}-\sum_{i=1}^{m} \ln \left[G\left(t_{i}\right)\right]\left\{W\left(t_{i} ; \theta\right)-1\right\}=0$,

where $W\left(t_{i} ; \theta\right)=\frac{R_{i}}{\left[G\left(t_{i}\right)\right]^{-\theta}-1}$. 
The Equation (13) is a nonlinear equation and requires the use of a numerical technique. For a given value of $t$, the ML estimators of RF and HRF can be obtained by the invariance property of ML estimations by replacing $\theta$ by $\hat{\theta}_{M L}$ in (9) and (10), respectively, with known $\underline{\eta}$.

\subsection{Case (II): $\theta$ and $\underline{\eta}$ are unknown}

In this case, $G(t ; \underline{\eta})$ in (7) is assumed to depend on an unknown $k$-dimensional vector $\underline{\eta}=\left(\eta_{1}, \eta_{2}, \ldots, \eta_{k}\right)$ of parameters. Therefore, $F(t ; \theta, \underline{\eta})$ depends on the unknown $k+1$ parameters $(\theta, \underline{\eta})$.

'Let $\underline{t}=\left(t_{1}, t_{2}, \ldots, t_{m}\right)$, with $t_{1} \leq t_{2} \leq \ldots \leq t_{m}$ denoting a progressive Type-II censored sample from $\operatorname{EGC}(\theta, \underline{\eta})$ and with $R_{1}, R_{2}, \ldots, R_{m}$ being the progressive censoring scheme. By substituting $F(t ; \theta, \underline{\eta})$ and $f(t ; \theta, \underline{\eta})$, which are given by (1) and (6), respectively, in (12) with $\Omega=(\theta, \underline{\eta})$, the LF based on the progressive Type II censored can be written as:

$$
L(\theta, \underline{\eta} ; \underline{t}) \propto \theta^{m} \prod_{i=1}^{m} \lambda^{\prime}\left(t_{i} ; \underline{\eta}\right) \exp \left(-\lambda\left(t_{i} ; \underline{\eta}\right)\right)\left[G\left(t_{i} ; \underline{\eta}\right)\right]^{\theta-1}\left\{1-\left[G\left(t_{i} ; \underline{\eta}\right)\right]^{\theta}\right\}^{R_{i}}
$$

where $G\left(t_{i} ; \underline{\eta}\right)$ is defined in (7).

Thus, to derive the MLEs of $\eta_{1}, \ldots, \eta_{k}$ and $\theta$, we have to find the partial derivative of the logarithm (15) and solve the system of the following nonlinear equations simultaneously with respect to $\eta_{1}, \ldots, \eta_{k}$ and $\theta$.

$$
\begin{aligned}
& \frac{m}{\theta}-\sum_{i=1}^{m} \ln \left[G\left(t_{i} ; \underline{\eta}\right)\right]\left\{W\left(t_{i} ; \theta, \underline{\eta}\right)-1\right\}=0, \\
& \sum_{i=1}^{m} \frac{\frac{\partial}{\partial \eta_{j}} \lambda^{\prime}\left(t_{i} ; \underline{\eta}\right)}{\lambda^{\prime}\left(t_{i} ; \underline{\eta}\right)}-\sum_{i=1}^{m} \frac{\partial}{\partial \eta_{j}} \lambda\left(t_{i} ; \underline{\eta}\right)\left\{1-\exp \left(-\lambda\left(t_{i} ; \underline{\eta}\right)\right)\left[\frac{\theta\left[1-W\left(t_{i} ; \theta, \underline{\eta}\right)\right]-1}{G\left(t_{i} ; \underline{\eta}\right)}\right]\right\}=0,
\end{aligned}
$$

where $W\left(t_{i} ; \theta, \underline{\eta}\right)$ is in (14) with $\underline{\eta}$ unknown. 
As it seems, the system of nonlinear equations (16) and (17) does not have an analytic solution in $\eta_{1}, \ldots, \eta_{k}$ and $\theta$. Therefore, a numerical method is needed to obtain the solution.

The invariance property of ML estimations can then be applied to obtain the ML estimators for $R\left(t_{0}\right)$ and $h\left(t_{0}\right)$, which are given in (9) and (10), respectively, for some $t_{0}$.

\section{Remarks}

The results obtained for the ML estimation for $\operatorname{EGC}(\theta, \underline{\eta})$ can be applied to other specified distributions, for example,

1- For an EP whose CDF is $F(t)=\left[1-(1+t)^{-\beta}\right]^{\theta}, \lambda(t ; \beta)=\beta \ln (1+t)$ and $G(t ; \beta)=1-(1+t)^{-\beta}$

* If $R_{1}=R_{2}=\ldots=R_{m}=0$ and $m=n$, then the ML estimations (16) and (17) coincide with those of Shawky and Abu-Zinadah (2009) in the case of a complete sample with $\eta_{j}=\lambda$.

* If $R_{1}=R_{2}=\ldots=R_{m-1}=0$ and $R_{m}=n-m$, then the ML estimations (16) and (17) coincide with those of Afify (2010) for a Type-II censored sample with $m=r, \eta_{j}=\alpha$, where $G\left(t_{m} ; \underline{\eta}\right)$ is defined in (7).

2- For an $\mathrm{EW}$ with $\operatorname{CDF} F(t ; \theta, \alpha)=\left[1-\exp \left(-t^{\alpha}\right)\right]^{\theta}, \lambda(t ; \alpha)=t^{\alpha}$ and $G(t ; \alpha)=1-\exp \left(-t^{\alpha}\right)$. If $\eta_{j}=\alpha$, then (16) and (17) agree with the result in Kim et al.(2009) for a progressive Type-II censored sample.

\section{Bayesian Estimation}

In this section, the parameters $\theta, \underline{\eta}, \mathrm{RF}$ and HRF at time $t_{0}$ are estimated based on a progressive Type-II censored sample from the EGC using Bayes methods on the following two cases 


\subsection{Case (I): $\theta$ is unknown and $\underline{\eta}$ is known}

Under the assumption that the parameter $\theta$ is unknown, supposing that the prior supposed by the experimenter is the general conjugate prior, which has been suggested by ALHussaini (1999) to be

$\pi(\theta ; \underline{\delta}) \propto C(\theta ; \underline{\delta}) \exp (-D(\theta ; \underline{\delta}))$

where $\underline{\delta}$ is a vector of hyperparameters.

The posterior density function of $\theta$ given the data, denoted by $\pi^{*}(\theta \mid \underline{t})$, after substituting with (12) and (18) is then given by

$\pi^{*}(\theta \mid \underline{t})=k \theta^{m} C(\theta ; \underline{\delta}) \exp \left\{-\left[D(\theta ; \underline{\delta})-\theta \sum_{i=1}^{m} \ln \left[G\left(t_{i}\right)\right]-\sum_{i=1}^{m} A\left(t_{i} ; \theta\right)\right]\right\}$,

where $A\left(t_{i} ; \theta\right)=R_{i} \ln \left\{1-\left[G\left(t_{i}\right)^{\theta}\right]\right\}$,

such that $k$ is a normalizing constant defined as

$k^{-1}=\int_{\theta} \theta^{m} C(\theta ; \underline{\delta}) \exp \left\{-\left[D(\theta ; \underline{\delta})-\theta \sum_{i=1}^{m} \ln \left[G\left(t_{i}\right)\right]-\sum_{i=1}^{m} A\left(t_{i} ; \theta\right)\right]\right\} d \theta$.

It is important to note that the posterior distribution does not simplify to nice closed forms. Therefore, numerical integration methods can be used.

Bayes estimators of any function of $\theta$, say $\phi(\theta)$, are obtained under two different types of loss functions as follows.

\section{Squared error loss function}

Under a SE loss function, the Bayes estimators of $\phi(\theta)$, denoted by $\hat{\phi}_{B S}(\theta)$, are obtained as follows:

$\hat{\phi}_{B S}(\theta)=k \int_{\theta} \phi(\theta) \theta^{m} C(\theta ; \underline{\delta}) \exp \left\{-\left[D(\theta ; \underline{\delta})-\theta \sum_{i=1}^{m} \ln \left[G\left(t_{i}\right)\right]-\sum_{i=1}^{m} A\left(t_{i} ; \theta\right)\right]\right\} d \theta$.

\section{LINEX loss function}


Under a LINEX loss function, the Bayes estimators of $\phi(\theta)$, denoted by $\hat{\phi}_{B L}(\theta)$, are obtained as follows:

$$
\hat{\phi}_{B L}(\theta)=-\frac{1}{a} \ln \left[k \int_{\theta} \theta^{m} C(\theta ; \underline{\delta}) \exp \left\{-\left[D(\theta ; \underline{\delta})-\theta \sum_{i=1}^{m} \ln \left[G\left(t_{i}\right)\right]-\sum_{i=1}^{m} A\left(t_{i} ; \theta\right)+a \phi(\theta)\right]\right\} d \theta\right] .
$$

\section{Remarks}

Using a non-informative prior, Bayes estimators can be found by substituting $\underline{\delta}$ with $\underline{0}$ in (18).

\subsection{Case (II): $\theta$ and $\underline{\eta}$ are unknown}

Under the assumption that the parameters $\theta$ and $\underline{\eta}=\eta_{1}, \ldots, \eta_{k}$ are unknown, suppose that $\theta$ and $\underline{\eta}$ are independent so that the prior supposed by the experimenter is given by $\pi(\theta, \underline{\eta})=\pi(\theta ; \underline{\delta}) \pi(\underline{\eta} ; \underline{\gamma})$

where $\underline{\delta}$ and $\underline{\gamma}$ are vectors of hyperparameters, $\pi(\theta ; \underline{\delta})$ is the same general conjugate prior given by (18) and $\pi(\underline{\eta} ; \underline{\delta})$ is the subjective prior. (Note that $\underline{\eta}=\eta_{1}, \ldots, \eta_{k}$, so $\pi(\underline{\eta} ; \underline{\gamma})$ is a vector of subjective priors). Then, the joint prior is

$\pi(\theta, \underline{\eta})=C(\theta ; \underline{\delta}) \exp (-D(\theta ; \underline{\delta})) \pi(\underline{\eta} ; \underline{\gamma})$.

The joint posterior density function of $\theta$ and $\underline{\eta}$ given the data, denoted by $\pi^{*}(\theta, \underline{\eta} \mid \underline{t})$, is found by substituting (15) and (22) with

$$
\pi^{*}(\theta, \underline{\eta} \mid \underline{t})=k \theta^{m} C(\theta ; \underline{\delta}) \pi(\underline{\eta} ; \underline{\gamma}) \times \exp \left\{-\left[\begin{array}{l}
D(\theta ; \underline{\delta})-\theta \sum_{i=1}^{m} \ln \left[G\left(t_{i} ; \underline{\eta}\right)\right]-\sum_{i=1}^{m} \ln \left[\lambda^{\prime}\left(t_{i} ; \underline{\eta}\right)\right]+ \\
\sum_{i=1}^{m} \lambda\left(t_{i} ; \underline{\eta}\right)-\sum_{i=1}^{m}\left(A\left(t_{i} ; \theta, \underline{\eta}\right)-\ln \left[G\left(t_{i} ; \underline{\eta}\right)\right]\right)
\end{array}\right]\right\},
$$

where $G\left(t_{i} ; \underline{\eta}\right)$ and $A\left(t_{i} ; \theta, \underline{\eta}\right)$ are defined in (7) and (19) with an unknown $\underline{\eta}$, 
such that $k$ is a normalizing constant defined as

$k^{-1}=\int_{\theta} \int_{\underline{\eta}} \theta^{m} C(\theta ; \underline{\delta}) \pi(\underline{\eta} ; \underline{\gamma}) \times \exp \left\{-\left[\begin{array}{l}D(\theta ; \underline{\delta})-\theta \sum_{i=1}^{m} \ln \left[G\left(t_{i} ; \underline{\eta}\right)\right]-\sum_{i=1}^{m} \ln \left[\lambda^{\prime}\left(t_{i} ; \underline{\eta}\right)\right]+ \\ \sum_{i=1}^{m} \lambda\left(t_{i} ; \underline{\eta}\right)-\sum_{i=1}^{m}\left(A\left(t_{i} ; \theta, \underline{\eta}\right)-\ln \left[G\left(t_{i} ; \underline{\eta}\right)\right]\right)\end{array}\right]\right\} d \underline{\eta} d \theta$,

where $\int_{\underline{\eta}} d \underline{\eta}=\int_{\eta_{1}} \int_{\eta_{2}} \ldots \int_{\eta_{k}} d \eta_{k} \ldots d \eta_{2} d \eta_{1}$.

Once again, we see that the posterior distribution does not simplify to nice closed forms.

Therefore, numerical methods are needed.

The Bayes estimators of any function of $\theta$ and $\eta_{j}(j=1, \ldots, k)$, say $\phi\left(\theta, \eta_{j}\right)$, are obtained under two different types of loss functions as follows.

\section{Squared error loss function}

Under a SE loss function, the Bayes estimator of $\phi\left(\theta, \eta_{j}\right)$, denoted by $\hat{\phi}_{B S}\left(\theta, \eta_{j}\right)$, is obtained as follows

$$
\hat{\phi}_{B S}\left(\theta, \eta_{j}\right)=k \int_{\theta} \int_{\underline{\eta}} \phi\left(\theta, \eta_{j}\right) \theta^{m} C(\theta ; \underline{\delta}) \pi(\underline{\eta} ; \underline{\gamma}) \times \exp \left\{-\left[\begin{array}{l}
D(\theta ; \underline{\delta})-\theta \sum_{i=1}^{m} \ln \left[G\left(t_{i} ; \underline{\eta}\right)\right]-\sum_{i=1}^{m} \ln \left[\lambda^{\prime}\left(t_{i} ; \underline{\eta}\right)\right]+ \\
\sum_{i=1}^{m} \lambda\left(t_{i} ; \underline{\eta}\right)-\sum_{i=1}^{m}\left(A\left(t_{i} ; \theta, \underline{\eta}\right)-\ln \left[G\left(t_{i} ; \underline{\eta}\right)\right]\right)
\end{array}\right]\right\} d \underline{\eta} d \theta
$$

\section{LINEX loss function}

Under a LINEX loss function, the Bayes estimator of $\phi\left(\theta, \eta_{j}\right)$, denoted by $\hat{\phi}_{B L}\left(\theta, \eta_{j}\right)$, is obtained as follows

$\hat{\phi}_{B L}\left(\theta, \eta_{j}\right)=-\frac{1}{a} \ln \left[k \int_{\theta} \int_{\underline{\eta}} \theta^{m} C(\theta ; \underline{\delta}) \pi(\underline{\eta} ; \underline{\gamma}) \times \exp \left\{-\left[\begin{array}{l}D(\theta ; \underline{\delta})-\theta \sum_{i=1}^{m} \ln \left[G\left(t_{i} ; \underline{\eta}\right)\right]-\sum_{i=1}^{m} \ln \left[\lambda^{\prime}\left(t_{i} ; \underline{\eta}\right)\right]+ \\ \sum_{i=1}^{m} \lambda\left(t_{i} ; \underline{\eta}\right)-\sum_{i=1}^{m}\left(A\left(t_{i} ; \theta, \underline{\eta}\right)-\ln \left[G\left(t_{i} ; \underline{\eta}\right)\right]+a \phi\left(\theta, \eta_{j}\right)\right.\end{array}\right]\right\} d \underline{\eta} d \theta\right]$.

\section{Remark}

Using a non-informative prior, Bayes estimators can be found by substituting $\underline{\delta}$ with $\underline{0}$ in (22). 


\section{Predictions of Times to Failure of Censored Units}

In this section, the problem of predicting times to failure of units censored in multiple stages $U_{S: R_{i}} ; s=1,2, \ldots, R_{i}, i=1,2, \ldots, m$ in the progressive censored sample $t_{1}, t_{2}, \ldots, t_{m}$ from the EGC is considered. The maximum likelihood prediction (MLP) of $U_{S: R_{i}}$ and the predictive maximum likelihood estimation (PMLE) of $\theta$ and $\underline{\eta}$ are conducted. Additionally, the Bayesian prediction of the unobserved failure times $U_{S: R_{i}}$ and the parameters $\theta$ and $\underline{\eta}$ are studied. Here, $U_{s: R_{i}},\left(s=1,2, \ldots, R_{i}\right)$ (defined as $U$ for short) denotes the $s^{\text {th }}$ order statistic of the $R_{i}$ removed units at the stages $i=1,2, \ldots, m$.

\subsection{Maximum likelihood prediction}

The MLP of times to failure of censored units and the PMLEs of the parameters are discussed for the following two cases.

\subsubsection{Case (I): $\theta$ is unknown and $\underline{\eta}$ is known}

The predictive likelihood function (PLF) of $U_{S: R_{i}}$ and $\Omega$ is given by (see, for example, Basak et al. (2006))

$$
\begin{array}{rr}
L\left(u_{S: R_{i}} ; \Omega \mid \underline{t}\right) \propto f\left(u_{s: R_{i}} ; \Omega\right)\left[F\left(u_{s: R_{i}} ; \Omega\right)-F\left(t_{i} ; \Omega\right)\right]^{s-1}\left[1-F\left(u_{s: R_{i}} ; \Omega\right)\right]^{R_{i}-s} \times \\
\prod_{k=1}^{m} f\left(t_{k} ; \Omega\right) \prod_{k=1, k \neq s}^{m}\left[1-F\left(t_{k} ; \Omega\right)\right]^{R_{k}}, & u_{s: R_{i}}>t_{i} .
\end{array}
$$

Then, the PLF of $U$ and $\theta$ for the given progressive censored sample $t_{1}, t_{2}, \ldots, t_{m}$ from the EGC can be obtained by substituting $F(t ; \theta, \underline{\eta}) \equiv F(t ; \theta)$ and $f(t ; \theta, \underline{\eta}) \equiv f(t ; \theta)$, which are given by (1) and (6), yielding 


$$
\begin{gathered}
L(u, \theta ; \underline{t}) \propto \theta^{m+1} \lambda^{\prime}(u) \exp (-\lambda(u))[G(u)]^{\theta-1}\left\{[G(u)]^{\theta}-\left[G\left(t_{i}\right)\right]^{\theta}\right\}^{s-1}\left\{1-[G(u)]^{\theta}\right\}^{R_{i}-s} \\
\prod_{k=1}^{m} \lambda^{\prime}\left(t_{k}\right) \exp \left(-\lambda\left(t_{k}\right)\right)\left[G\left(t_{k}\right)\right]^{\theta-1} \prod_{k=1, k \neq i}^{m}\left\{1-\left[G\left(t_{k}\right)\right]^{\theta}\right\}^{R_{k}}, u>t_{i} .
\end{gathered}
$$

Then, the predictive likelihood equations for $\theta$ and $u$ are given by differentiating the logarithm of (26) with respect to $\theta$ and $u$, respectively, as follows:

$$
\begin{aligned}
& \frac{m+1}{\theta}+\ln [G(u)]\left\{1+[G(u)]^{\theta}[V(u, \theta)-Z(u, \theta)]\right\}-\sum_{k=1}^{m} \ln \left[G\left(t_{k}\right)\right]\left\{W\left(t_{k} ; \theta\right)-1\right\}- \\
& {\left[G\left(t_{i}\right)\right]^{\theta} \ln \left[G\left(t_{i}\right)\right]\left\{V(u, \theta)-\left[G\left(t_{i}\right)\right]^{-\theta} W\left(t_{i} ; \theta\right)\right\}=0,} \\
& \frac{\lambda^{\prime \prime}(u)}{\lambda^{\prime}(u)}-\lambda^{\prime}(u)\left\{1+\frac{\exp (-\lambda(u))}{G(u)}\left[1-\theta\left[1+[G(u)]^{\theta}[V(u, \theta)-Z(u, \theta)]\right]\right\}=0,\right.
\end{aligned}
$$

where $W(. . ; \theta)$ is defined in (15),

$$
V(u, \theta)=\frac{s-1}{[G(u)]^{\theta}-\left[G\left(t_{i}\right)\right]^{\theta}} \quad \text { and } \quad Z(u, \theta)=\frac{R_{i}-s}{1-[G(u)]^{\theta}} .
$$

Then, (27) and (28) are solved simultaneously to find the PMLE of $\theta$, which is $\hat{\theta}_{P M L}$. Subsequently, by substituting $\hat{\theta}_{P M L}$ in (28), the MLP of $u$ is obtained.

\subsubsection{Case (II): $\theta$ and $\underline{\eta}$ are unknown}

After substituting for $F(t ; \theta, \underline{\eta})$ and $f(t ; \theta, \underline{\eta})$, which are given by (1) and (6), respectively, in (26) with $\Omega=(\theta, \underline{\eta})$, the PLF of $U_{S: R_{i}}, \theta$ and $\underline{\eta}$ for the given progressive censored sample $t_{1}, t_{2}, \ldots, t_{m}$ from the EGC can be written as

$$
\begin{gathered}
L(u, \theta, \underline{\eta}, \underline{t}) \propto \theta^{m+1} \lambda^{\prime}(u, \underline{\eta}) \exp (-\lambda(u, \underline{\eta}))[G(u, \underline{\eta})]^{\theta-1}\left\{[G(u, \underline{\eta})]^{\theta}-\left[G\left(t_{i}, \underline{\eta}\right)\right]^{\theta}\right\}^{s-1}\left\{1-[G(u, \underline{\eta})]^{\theta}\right\}^{R_{i}-s} \\
\times \prod_{k=1}^{m} \lambda^{\prime}\left(t_{k}, \underline{\eta}\right) \exp \left(-\lambda\left(t_{k}, \underline{\eta}\right)\right)\left[G\left(t_{k}, \underline{\eta}\right)\right]^{\theta-1} \prod_{k=1, k \neq i}^{m}\left\{1-\left[G\left(t_{k}, \underline{\eta}\right)\right]^{\theta}\right\}^{R_{k}} \quad, \quad u \geq t_{i} .
\end{gathered}
$$


Then, the predictive likelihood equations for $\theta, u$ and $\eta_{j}$ are given from the differentials of the logarithm of (30) with respect to $\theta, u$ and $\eta_{j}$, respectively, as follows:

$$
\begin{aligned}
& \frac{m+1}{\theta}+\ln [G(u ; \underline{\eta})]\left\{1+[G(u, \underline{\eta})]^{\theta}[V(u, \theta, \underline{\eta})-Z(u, \theta, \underline{\eta})]\right\}-\sum_{k=1}^{m} \ln G\left(t_{k}, \underline{\eta}\right)\left\{W\left(t_{k} ; \theta, \underline{\eta}\right)-1\right\} \\
& -\left[G\left(t_{i}, \underline{\eta}\right)\right]^{\theta} \ln \left[G\left(t_{i}, \underline{\eta}\right)\right]\left\{V(u, \theta, \underline{\eta})-\left[G\left(t_{i}, \underline{\eta}\right)\right]^{-\theta} W\left(t_{i} ; \theta, \underline{\eta}\right)\right\}=0, \\
& \frac{\lambda^{\prime \prime}(u, \underline{\eta})}{\lambda^{\prime}(u, \underline{\eta})}-\lambda^{\prime}(u, \underline{\eta})\left[1+\frac{\exp (-\lambda(u, \underline{\eta}))}{G(u, \underline{\eta})}\left[1-\theta\left\{1+[G(u, \underline{\eta})]^{\theta}[V(u, \theta, \underline{\eta})-Z(u, \theta, \underline{\eta})]\right]\right]=0,\right. \\
& \frac{1}{\lambda^{\prime}(u, \underline{\eta})} \frac{\partial}{\partial \eta_{j}} \lambda^{\prime}(u, \underline{\eta})+\sum_{k=1}^{m} \frac{1}{\lambda^{\prime}\left(t_{k}, \underline{\eta}\right)} \frac{\partial}{\partial \eta_{j}} \lambda^{\prime}\left(t_{k}, \underline{\eta}\right)- \\
& \frac{\partial}{\partial \eta_{j}} \lambda(u, \underline{\eta})\left\{1+\frac{\exp (-\lambda(u, \underline{\eta}))}{G(u, \underline{\eta})}\left[1-\theta\left\{1+[G(u, \underline{\eta})]^{\theta}[V(u, \theta, \underline{\eta})-Z(u, \theta, \underline{\eta})]\right\}\right]+\right. \\
& \sum_{k=1}^{m} \frac{\partial}{\partial \eta_{j}} \lambda\left(t_{k}, \underline{\eta}\right)\left\{\frac{\exp \left(-\lambda\left(t_{k}, \underline{\eta}\right)\right)}{G\left(t_{k}, \underline{\eta}\right)}\left[\theta\left(1-W\left(t_{k} ; \theta, \underline{\eta}\right)\right)-1\right]-1\right\}- \\
& \theta\left[G\left(t_{i}, \underline{\eta}\right)\right]^{\theta-1} \exp \left(-\lambda\left(t_{i}, \underline{\eta}\right)\right) \frac{\partial}{\partial \eta_{j}} \lambda\left(t_{i}, \underline{\eta}\right)\left\{V(u, \theta, \underline{\eta})-\left[G\left(t_{i}, \underline{\eta}\right)\right]^{\theta} W\left(t_{i} ; \theta, \underline{\eta}\right)\right\}=0,
\end{aligned}
$$

where $G(. ., \underline{\eta}), W(. . ; \theta, \underline{\eta}), V(u, \theta, \underline{\eta})$ and $Z(u, \theta, \underline{\eta})$ are defined in (7), (14) and (29), respectively, with $\underline{\eta}$ unknown. Then, (31), (32) and (33) are solved simultaneously to find the PMLEs of $\theta$ and $\eta_{j}$, which are $\hat{\theta}_{P M L}$ and $\hat{\eta}_{j_{P M L}}$, respectively. Subsequently, by substituting $\hat{\theta}_{P M L}$ and $\hat{\eta}_{j_{P M L}}$ in (32), the MLP of $u$ is obtained.

\subsection{Bayesian prediction}

The Bayesian prediction of times to failure of censored units and the Bayesian prediction estimates of the parameters are conducted for the following two cases.

\subsubsection{Case (I): $\theta$ is unknown and $\underline{\eta}$ is known}


By forming the product of the PLF in (26) and the prior in (18), we obtain the joint predictive likelihood (see, for example, Madi and Raqab (2009)) to be

$$
\begin{aligned}
& \pi^{*}(u, \theta \mid \underline{t})=k \theta^{m+1} C(\theta ; \underline{\delta}) \frac{\lambda^{\prime}(u) \exp (-\lambda(u))}{G(u)}\left\{1-\left[G\left(t_{i}\right)\right]^{\theta}\right\}^{-R_{i}} \times \\
& \sum_{j=0}^{s-1} \sum_{l=0}^{R_{i}-s} Q \exp \left\{-\left[\begin{array}{l}
\left.D(\theta ; \underline{\delta})-\theta\left[(s-j+l) \ln [G(u)]+j \ln \left[G\left(t_{i}\right)\right]+\sum_{k=1}^{m} \ln \left[G\left(t_{k}\right)\right]\right]-\right] \\
\sum_{k=1}^{m} A\left(t_{k} ; \theta\right)
\end{array}\right]\right\},
\end{aligned}
$$

where $Q=\left(\begin{array}{c}s-1 \\ j\end{array}\right)\left(\begin{array}{c}R_{i}-s \\ l\end{array}\right)(-1)^{j+l}$,

and $A(. . ; \theta)$ is defined in $(20)$,

such that $k$ is a normalizing constant defined as

$$
\begin{aligned}
k^{-1}= & \sum_{j=0}^{s-1} \sum_{l=0}^{R_{i}-s} \frac{Q}{s-j+l} \int_{\theta} \theta^{m} C(\theta ; \underline{\delta})\left\{1-\left[G\left(t_{i}\right)\right]^{\theta}\right\}^{-R_{i}}\left[1-\exp \left\{\theta(s-j+l) \ln \left[G\left(t_{i}\right)\right]\right\}\right] \times \\
& \exp \left\{-\left[D(\theta ; \underline{\delta})-\theta\left[j \ln \left[G\left(t_{i}\right)\right]+\sum_{k=1}^{m} \ln \left[G\left(t_{k}\right)\right]\right]-\sum_{k=1}^{m} A\left(t_{k} ; \theta\right)\right]\right\} d \theta .
\end{aligned}
$$

The Bayes prediction is obtained under two different loss functions as follows:

\section{Squared error loss function}

Under a SE loss function, the Bayes predictions can be obtained as follows:

- The Bayes prediction of $U_{S: R_{i}}$, denoted by $\hat{U}_{B S}$, is

$$
\begin{aligned}
\hat{U}_{B S}= & k \sum_{j=0}^{s-1} \sum_{l=0}^{R_{i}-s} Q \int_{\theta} \int_{t_{i}}^{\infty} u \theta^{m+1} C(\theta ; \underline{\delta}) \frac{\lambda^{\prime}(u) \exp (-\lambda(u))}{G(u)}\left\{1-\left[G\left(t_{i}\right)\right]^{\theta}\right\}^{-R_{i}} \times \\
& \exp \left\{-\left[D(\theta ; \underline{\delta})-\theta\left[(s-j+l) \ln [G(u)]+j \ln \left[G\left(t_{i}\right)\right]+\sum_{k=1}^{m} \ln \left[G\left(t_{k}\right)\right]\right]-\sum_{k=1}^{m} A\left(t_{k} ; \theta\right)\right]\right\} d u d \theta .
\end{aligned}
$$

- The Bayes predictive estimate of $\theta$, denoted by $\hat{\theta}_{B S}$, is 


$$
\begin{aligned}
\hat{\theta}_{B S} & =k \sum_{j=0}^{s-1} \sum_{l=0}^{R_{i}-s} Q \int_{\theta} \int_{t_{i}}^{\infty} \theta^{m+2} C(\theta ; \underline{\delta}) \frac{\lambda^{\prime}(u) \exp (-\lambda(u))}{G(u)}\left\{1-\left[G\left(t_{i}\right)\right]^{\theta}\right\}^{-R_{i}} \times \\
& \exp \left\{-\left[D(\theta ; \underline{\delta})-\theta\left[(s-j+l) \ln [G(u)]+j \ln \left[G\left(t_{i}\right)\right]+\sum_{k=1}^{m} \ln \left[G\left(t_{k}\right)\right]\right]-\sum_{k=1}^{m} A\left(t_{k} ; \theta\right)\right]\right\} d u d \theta .
\end{aligned}
$$

\section{LINEX loss function}

Under a LINEX loss function, the Bayes predictions can be obtained as follows:

- The Bayes prediction of $U_{S: R_{i}}$, denoted by $\hat{U}_{B L}$, is

$$
\hat{U}_{B L}=-\frac{1}{a} \ln \left[\begin{array}{c}
k \sum_{j=0}^{s-1} \sum_{l=0}^{R_{i}-s} Q \int_{\theta} \int_{t_{i}}^{\infty} \theta^{m+1} C(\theta ; \underline{\delta}) \frac{\lambda^{\prime}(u) \exp (-\lambda(u))}{G(u)}\left\{1-\left[G\left(t_{i}\right)\right]^{\theta}\right\}^{-R_{i}} \\
\left.\exp \left\{-\left[\begin{array}{l}
\left.D(\theta ; \underline{\delta})-\theta\left[(s-j+l) \ln [G(u)]+j \ln \left[G\left(t_{i}\right)\right]+\sum_{k=1}^{m} \ln \left[G\left(t_{k}\right)\right]\right]-\right] \\
\sum_{k=1}^{m} A\left(t_{k} ; \theta\right)+a u
\end{array}\right]\right\} d u d \theta\right] .
\end{array}\right.
$$

- The Bayes predictive estimate of $\theta$, denoted by $\hat{\theta}_{B L}$, is

$$
\hat{\theta}_{B L}=-\frac{1}{a} \ln \left[\begin{array}{l}
k \sum_{j=0}^{s-1} \sum_{l=0}^{R_{i}-s} Q \int_{\theta} \int_{t_{i}}^{\infty} \theta^{m+1} C(\theta ; \underline{\delta}) \frac{\lambda^{\prime}(u) \exp (-\lambda(u))}{G(u)}\left\{1-\left[G\left(t_{i}\right)\right]^{\theta}\right\}^{-R_{i}} \\
\left.\exp \left\{-\left[\begin{array}{l}
D(\theta ; \underline{\delta})-\theta\left[(s-j+l) \ln [G(u)]+j \ln \left[G\left(t_{i}\right)\right]+\sum_{k=1}^{m} \ln \left[G\left(t_{k}\right)\right]-a\right] \\
-\sum_{k=1}^{m} A\left(t_{k} ; \theta\right)
\end{array}\right]\right\} d u d \theta\right] .
\end{array}\right] .
$$

where $G(.),. A(. . ; \theta), Q, k^{-1}$ are defined in (7), (20), (35) and (36), respectively.

\subsubsection{Case (II): $\theta$ and $\underline{\eta}$ are unknown}


By forming the product of the PLF in (30) and the joint priors of $\theta$ and $\underline{\eta}$ in (22), we obtain the joint predictive likelihood to be

$$
\begin{gathered}
\pi^{*}(u, \theta, \underline{\eta} \mid \underline{t})=k \theta^{m+1} C(\theta ; \underline{\delta}) \frac{\lambda^{\prime}(u ; \underline{\eta}) \exp (-\lambda(u ; \underline{\eta}))}{G(u ; \underline{\eta})} \pi(\underline{\eta} ; \underline{\gamma})\left\{1-\left[G\left(t_{i} ; \underline{\eta}\right)\right]^{\theta}\right\}^{-R_{i}} \sum_{j=0}^{s-1} \sum_{l=0}^{R_{i}-s} Q \times \\
\exp \left\{-\left[\begin{array}{l}
\left.\left.D(\theta ; \underline{\delta})-\theta\left[(s-j+l) \ln [G(u ; \underline{\eta})]+j \ln \left[G\left(t_{i} ; \underline{\eta}\right)\right]+\sum_{k=1}^{m} \ln \left[G\left(t_{k} ; \underline{\eta}\right)\right]\right]-\right]\right) \\
\sum_{k=1}^{m} \ln \left[\lambda^{\prime}\left(t_{k} ; \underline{\eta}\right)\right]+\sum_{k=1}^{m} \lambda\left(t_{k} ; \underline{\eta}\right)-\sum_{k=1}^{m}\left(A\left(t_{k} ; \theta, \underline{\eta}\right)-\ln \left[G\left(t_{k} ; \underline{\eta}\right)\right]\right)
\end{array}\right]\right\},
\end{gathered}
$$

where $Q$ and $A(. . ; \theta, \underline{\eta})$ are defined in (35) and (20), respectively, with $\underline{\eta}$ unknown, such that $k$ is a normalizing constant defined as

$$
\begin{aligned}
k^{-1}=\sum_{j=0}^{s-1} \sum_{l=0}^{R_{i}-s} \frac{Q}{s-j+l} \int_{\theta} \int_{\underline{\eta}} \theta^{m} C(\theta ; \underline{\delta}) \pi(\underline{\eta} ; \underline{\gamma})\left\{1-\left[G\left(t_{i} ; \underline{\eta}\right)\right]^{\theta}\right\}^{-R_{i}}\left[1-\exp \left\{\theta(s-j+l) \ln \left[G\left(t_{i} ; \underline{\eta}\right)\right]\right\}\right] \\
\times \exp \left\{\begin{array}{l}
{\left[D(\theta ; \underline{\delta})-\theta\left[\left(j \ln \left[G\left(t_{i} ; \underline{\eta}\right)\right]+\sum_{k=1}^{m} \ln \left[G\left(t_{k} ; \underline{\eta}\right)\right]\right]-\right.\right.} \\
\left.\left[\sum_{k=1}^{m} \ln \left[\lambda^{\prime}\left(t_{k} ; \underline{\eta}\right)\right]+\sum_{k=1}^{m} \lambda\left(t_{k} ; \underline{\eta}\right)-\sum_{k=1}^{m}\left(A\left(t_{k} ; \theta, \underline{\eta}\right)-\ln \left[G\left(t_{k} ; \underline{\eta}\right)\right]\right)\right]\right\} d \underline{\eta} d \theta .
\end{array}\right.
\end{aligned}
$$

The Bayesian predictions are obtained under two different loss functions as follows:

\section{Squared error loss function}

Under a SE loss function, the Bayes predictions are as follows:

- The Bayes prediction of $U_{S: R_{i}}$, denoted by $\hat{U}_{B S}$, is

$$
\begin{aligned}
& \hat{U}_{B S}= k \sum_{j=0}^{s-1} \sum_{l=0}^{R_{i}-s} Q \int_{\theta} \int_{\underline{\eta}} \int_{t_{i}}^{\infty} u \theta^{m+1} C(\theta ; \underline{\delta}) \frac{\lambda^{\prime}(u ; \underline{\eta}) \exp (-\lambda(u ; \underline{\eta}))}{G(u ; \underline{\eta})} \pi(\underline{\eta} ; \underline{\gamma})\left\{1-\left[G\left(t_{i} ; \underline{\eta}\right)\right]^{\theta}\right\}^{-R_{i}} \times \\
& \exp \left\{-\left[\begin{array}{l}
\left.D(\theta ; \underline{\delta})-\theta\left[(s-j+l) \ln [G(u ; \underline{\eta})]+j \ln \left[G\left(t_{i} ; \underline{\eta}\right)\right]+\sum_{k=1}^{m} \ln \left[G\left(t_{k} ; \underline{\eta}\right)\right]\right]-\right] \\
\sum_{k=1}^{m} \ln \left[\lambda^{\prime}\left(t_{k} ; \underline{\eta}\right)\right]+\sum_{k=1}^{m} \lambda\left(t_{k} ; \underline{\eta}\right)-\sum_{k=1}^{m}\left(A\left(t_{k} ; \theta, \underline{\eta}\right)-\ln \left[G\left(t_{k} ; \underline{\eta}\right)\right]\right)
\end{array}\right] d u d \underline{\eta} \underline{d \theta .}\right.
\end{aligned}
$$

- The Bayes predictive estimate of $\theta$, denoted by $\hat{\theta}_{B S}$, is 


$$
\begin{aligned}
& \hat{\theta}_{B S}= k \sum_{j=0}^{s-1} \sum_{l=0}^{R_{i}-s} Q \int_{\theta} \int_{\underline{\eta}} \int_{t_{i}}^{\infty} \theta^{m+2} C(\theta ; \underline{\delta}) \frac{\lambda^{\prime}(u ; \underline{\eta}) \exp (-\lambda(u ; \underline{\eta}))}{G(u ; \underline{\eta})} \pi(\underline{\eta} ; \underline{\gamma})\left\{1-\left[G\left(t_{i} ; \underline{\eta}\right)\right]^{\theta}\right\}^{-R_{i}} \times \\
& \exp \left\{-\left[\begin{array}{l}
\left.\left.D(\theta ; \underline{\delta})-\theta\left[(s-j+l) \ln [G(u ; \underline{\eta})]+j \ln \left[G\left(t_{i} ; \underline{\eta}\right)\right]+\sum_{k=1}^{m} \ln \left[G\left(t_{k} ; \underline{\eta}\right)\right]\right]-\right]\right\} d u d \underline{\eta} d \theta \\
\sum_{k=1}^{m} \ln \left[\lambda^{\prime}\left(t_{k} ; \underline{\eta}\right)\right]+\sum_{k=1}^{m} \lambda\left(t_{k} ; \underline{\eta}\right)-\sum_{k=1}^{m}\left(A\left(t_{k} ; \theta, \underline{\eta}\right)-\ln \left[G\left(t_{k} ; \underline{\eta}\right)\right]\right)
\end{array}\right]\right\}
\end{aligned}
$$

- The Bayes predictive estimate of $\eta_{j}$, denoted by $\hat{\eta}_{j_{B S}}$, is

$$
\begin{aligned}
& \hat{\eta}_{j_{B S}}= k \sum_{j=0}^{s-1} \sum_{l=0}^{R_{i}-s} Q \int_{\theta} \int_{\underline{\eta}} \int_{t_{i}}^{\infty} \eta_{j} \theta^{m+1} C(\theta ; \underline{\delta}) \frac{\lambda^{\prime}(u ; \underline{\eta}) \exp (-\lambda(u ; \underline{\eta}))}{G(u ; \underline{\eta})} \pi(\underline{\eta} ; \underline{\gamma})\left\{1-\left[G\left(t_{i} ; \underline{\underline{\eta}}\right)\right]^{\theta}\right\}^{-R_{i}} \times \\
& \exp \left\{-\left[\begin{array}{l}
\left.\left.D(\theta ; \underline{\delta})-\theta\left[(s-j+l) \ln [G(u ; \underline{\eta})]+j \ln \left[G\left(t_{i} ; \underline{\eta}\right)\right]+\sum_{k=1}^{m} \ln \left[G\left(t_{k} ; \underline{\eta}\right)\right]\right]-\right]\right) \\
\sum_{k=1}^{m} \ln \left[\lambda^{\prime}\left(t_{k} ; \underline{\eta}\right)\right]+\sum_{k=1}^{m} \lambda\left(t_{k} ; \underline{\eta}\right)-\sum_{k=1}^{m}\left(A\left(t_{k} ; \theta, \underline{\eta}\right)-\ln \left[G\left(t_{k} ; \underline{\eta}\right)\right]\right)
\end{array}\right]\right\} d \underline{\eta} \underline{d} d \theta .
\end{aligned}
$$

\section{LINEX loss function}

Under a LINEX loss function, the Bayes predictions can be obtained as follows.

- The Bayes predictive prediction of $U_{S: R_{i}}$, denoted by $\hat{U}_{B L}$, is

$$
\hat{U}_{B S}=-\frac{1}{a} \ln \left[\begin{array}{l}
k \sum_{j=0}^{s-1} \sum_{l=0}^{R_{i}-s} Q \int_{\theta} \int_{\underline{\eta}} \int_{t_{i}}^{\infty} \theta^{m+1} C(\theta ; \underline{\delta}) \frac{\lambda^{\prime}(u ; \underline{\eta}) \exp (-\lambda(u ; \underline{\eta}))}{G(u ; \underline{\eta})} \pi(\underline{\eta} ; \underline{\gamma})\left\{1-\left[G\left(t_{i} ; \underline{\eta}\right)\right]^{\theta}\right\}^{-R_{i}} \times \\
\left.\exp \left\{-\left[\begin{array}{l}
D(\theta ; \underline{\delta})-\theta\left[(s-j+l) \ln [G(u ; \underline{\eta})]+j \ln \left[G\left(t_{i} ; \underline{\eta}\right)\right]+\sum_{k=1}^{m} \ln \left[G\left(t_{k} ; \underline{\eta}\right)\right]\right]- \\
\sum_{k=1}^{m} \ln \left[\lambda^{\prime}\left(t_{k} ; \underline{\eta}\right)\right]+\sum_{k=1}^{m} \lambda\left(t_{k} ; \underline{\eta}\right)-\sum_{k=1}^{m}\left(A\left(t_{k} ; \theta, \underline{\eta}\right)-\ln \left[G\left(t_{k} ; \underline{\eta}\right)\right]\right)+a u
\end{array}\right]\right\} d u d \underline{\eta} d \theta\right] . \\
\end{array}\right] .
$$

- The Bayes predictive estimate of $\theta$, denoted by $\hat{\theta}_{B L}$, is 


$$
\hat{\theta}_{B L}=-\frac{1}{a} \ln \left[\begin{array}{l}
k \sum_{j=0}^{s-1} \sum_{l=0}^{R_{i}-s} Q \int_{\theta} \int_{\underline{\eta}} \int_{t_{t}}^{\infty} \theta^{m+1} C(\theta ; \underline{\delta}) \frac{\lambda^{\prime}(u ; \underline{\eta}) \exp (-\lambda(u ; \underline{\eta}))}{G(u ; \underline{\eta})} \pi(\underline{\eta} ; \underline{\gamma})\left\{1-\left[G\left(t_{i} ; \underline{\eta}\right)\right]^{\theta}\right\}^{-R_{i}} \times \\
\left.\exp \left\{-\left[\begin{array}{l}
\left.D(\theta ; \underline{\delta})-\theta\left[(s-j+l) \ln [G(u ; \underline{\eta})]+j \ln \left[G\left(t_{i} ; \underline{\eta}\right)\right]+\sum_{k=1}^{m} \ln \left[G\left(t_{k} ; \underline{\eta}\right)\right]-a\right]-\right] \\
\sum_{k=1}^{m} \ln \left[\lambda^{\prime}\left(t_{k} ; \underline{\eta}\right)\right]+\sum_{k=1}^{m} \lambda\left(t_{k} ; \underline{\eta}\right)-\sum_{k=1}^{m}\left(A\left(t_{k} ; \theta, \underline{\eta}\right)-\ln \left[G\left(t_{k} ; \underline{\eta}\right)\right]\right)
\end{array}\right]\right\} d u d \theta d \underline{\eta} \underline{\eta}\right)
\end{array} .\right.
$$

- The Bayes predictive estimate of $\eta_{j}$, denoted by $\hat{\eta}_{j_{B L}}$, is

$$
\hat{\eta}_{j_{B L}}=-\frac{1}{a} \ln \left[\begin{array}{c}
k \sum_{j=0}^{s-1} \sum_{l=0}^{R_{i}-s} Q \int_{\theta} \int_{\underline{\eta}} \int_{t_{i}}^{\infty} \theta^{m+1} C(\theta ; \underline{\delta}) \frac{\lambda^{\prime}(u ; \underline{\eta}) \exp (-\lambda(u ; \underline{\eta}))}{G(u ; \underline{\eta})} \pi(\underline{\eta} ; \underline{\gamma})\left\{1-\left[G\left(t_{i} ; \underline{\eta}\right)\right]^{\theta}\right\}^{-R_{i}} \times \\
\left.\exp \left\{-\left[\begin{array}{l}
\left.D(\theta ; \underline{\delta})-\theta\left[(s-j+l) \ln [G(u ; \underline{\eta})]+j \ln \left[G\left(t_{i} ; \underline{\eta}\right)\right]+\sum_{k=1}^{m} \ln \left[G\left(t_{k} ; \underline{\eta}\right)\right]\right]-\right] \\
\sum_{k=1}^{m} \ln \left[\lambda^{\prime}\left(t_{k} ; \underline{\eta}\right)\right]+\sum_{k=1}^{m} \lambda\left(t_{k} ; \underline{\eta}\right)-\sum_{k=1}^{m}\left(A\left(t_{k} ; \theta, \underline{\eta}\right)-\ln \left[G\left(t_{k} ; \underline{\eta}\right)\right]\right)+a \eta \eta_{j}
\end{array}\right]\right\} d u d \underline{\eta} d \theta\right]
\end{array}\right],
$$

where $G(. . ; \underline{\eta}), A(. . ; \theta, \underline{\eta}), Q$, and $k^{-1}$ are defined in (7), (20) with $\underline{\eta}$ unknown, (35), and (36).

\section{Two-sample prediction}

In this section, a two-sample scheme is used in which the informative sample is a progressive Type-II censored sample, and $Y_{1} \leq Y_{2} \leq \ldots \leq Y_{N_{f}}$ is the order statistics of a future sample. It is assumed that the two samples are independent, and each of their corresponding random samples is obtained from an EGC population with CDF (6). Therefore, $Y_{s}$ is the $s^{\text {th }}$ ordered statistic in the future sample of size $N_{f}, 1<s<N_{f}$ with lifetimes distributed as (5).

\subsection{Case (I): $\theta$ is unknown and $\underline{\eta}$ is known}

For the given values of the parameter $\Omega$, the PDF of the $Y_{s}, s=1,2, \ldots, N_{f} \quad$ (see, for example, Ali Mousa and Jaheen (2002) and Basak et al. (2009)) is given by 
$h\left(y_{s} \mid \Omega\right)=D(s)\left[1-F_{T}\left(y_{s} \mid \Omega\right)\right]^{N-s}\left[F_{T}\left(y_{s} \mid \Omega\right)\right]^{s-1} f_{T}\left(y_{s} \mid \Omega\right)$

where, $D(s)=s\left(\begin{array}{l}N_{f} \\ s\end{array}\right)$.

Substituting (6) and (7) in (50) with $\Omega=\theta$, the PDF of $Y_{s}, s=1,2, \ldots, N_{f}$, is

$h\left(y_{s} \mid \theta\right)=D(s) \theta \frac{\lambda^{\prime}\left(y_{s}\right) \exp \left(\lambda\left(y_{s}\right)\right)}{G\left(y_{s}\right)} \sum_{j=0}^{N_{f}-s} Q \exp \left\{\theta(s+j) \ln \left[G\left(y_{s}\right)\right]\right\}$,

where $Q=\left(\begin{array}{c}N_{f}-s \\ j\end{array}\right)(-1)^{j}$

The Bayes predictive density of $Y_{s}$ is the product of posterior (19) and the PDF of $Y_{s}$ in (51) integrated over $\theta$ as follows:

$$
\begin{aligned}
h^{*}\left(y_{s} \mid \underline{t}\right) & =D(s) k \frac{\lambda^{\prime}\left(y_{s}\right) \exp \left(\lambda\left(y_{s}\right)\right)}{G\left(y_{s}\right)} \sum_{h=0}^{N_{f}-s} Q \int_{\theta} \theta^{m+1} C(\theta ; \underline{\delta}) \times \\
& \exp \left\{-\left[D(\theta ; \underline{\delta})-\theta\left\{(s+j) \ln \left[G\left(y_{s}\right)\right]+\sum_{i=1}^{m} \ln \left[G\left(t_{i}\right)\right]\right\}-\sum_{i=1}^{m} A\left(t_{i} ; \theta\right)\right]\right\} d \theta,
\end{aligned}
$$

where $D(s)$ is defined in (45), $k^{-1}$ is defined in (21), $Q$ is defined in (46), $C(\theta ; \underline{\delta})$ and $D(\theta ; \underline{\delta})$ are defined in (18), $G\left(y_{s}\right)$, and $G^{\prime}\left(y_{s}\right)$ and $A\left(t_{i} ; \theta\right)$ are defined in (7) and (20), respectively.

It is apparent that $h^{*}\left(y_{s} \mid \underline{t}\right)$ cannot be expressed in closed form, and hence, it can be evaluated numerically. The Bayesian prediction bounds for $Y_{s}$ can be found through the survival function of $Y_{s}$ as $P\left(Y_{s} \geq v\right)=\int_{v}^{\infty} h^{*}\left(y_{s} \mid \underline{t}\right) d y_{s}$ for some positive value of $v$. Having obtained $P\left(Y_{s} \geq v \mid \underline{t}\right)$, a $100 \tau \%$ Bayesian predictive interval for $Y_{s}$ is then given by $P\left[L L \leq Y_{s} \leq U L\right]=\tau$, where the lower limit $(L L)$ and upper limit $(U L)$ of the $100 \tau \%$ Bayesian predictive interval for $Y_{s}$ are obtained by solving these two equations, $P\left(Y_{s} \geq L L \mid \underline{t}\right)=\frac{1+\tau}{2} \quad$ and $\quad P\left(Y_{s} \geq U L \mid \underline{t}\right)=\frac{1-\tau}{2}$. 


\subsection{Case (II): $\theta$ and $\underline{\eta}$ are unknown}

Substituting (5) and (7) in (49) with $\Omega=(\theta, \underline{\eta})$, we obtain the PDF of $Y_{s}, s=1,2, \ldots, N_{f}$

, as

$h\left(y_{s} \mid \theta, \underline{\eta}\right)=D(s) \theta \frac{\lambda^{\prime}\left(y_{s} ; \underline{\eta}\right) \exp \left(\lambda\left(y_{s} ; \underline{\eta}\right)\right)}{G\left(y_{s} ; \underline{\eta}\right)} \sum_{j=0}^{N_{f}-s} Q \exp \left\{\theta(j+s) \ln \left[G\left(y_{s} ; \underline{\eta}\right)\right]\right\}$,

where $Q$ is defined in (52).

The Bayes predictive density of $Y_{s}$ is obtained as the product of posterior (23) and the PDF of $Y_{s}$ in (48) and integrating the $k+1$ integral out of $\theta$ and $\underline{\eta}=\left(\eta_{1}, \eta_{2}, \ldots, \eta_{k}\right)$ as follows

$$
\begin{aligned}
& h^{*}\left(y_{s} \mid \underline{t}\right)=D(s) k \sum_{h=0}^{N_{f}-s} Q \int_{\theta} \int_{\underline{\eta}} \theta^{m+1} C(\theta ; \underline{\delta}) \pi(\underline{\eta} ; \underline{\gamma}) \frac{\lambda^{\prime}\left(y_{s} ; \underline{\eta}\right) \exp \left(\lambda\left(y_{s} ; \underline{\eta}\right)\right)}{G\left(y_{s} ; \underline{\eta}\right)} \times \\
& \exp \left\{-\left[\begin{array}{l}
D(\theta ; \underline{\delta})-\theta\left\{(s+j) \ln \left[G\left(y_{s} ; \underline{\eta}\right)\right]+\sum_{i=1}^{m} \ln \left[G\left(t_{i} ; \underline{\eta}\right)\right]\right\}- \\
\left.\left.\sum_{i=1}^{m} \ln \left[\lambda^{\prime}\left(t_{i} ; \underline{\eta}\right)\right]+\sum_{i=1}^{m} \lambda\left(t_{i} ; \underline{\eta}\right)-\sum_{i=1}^{m}\left(A\left(t_{i} ; \theta, \underline{\eta}\right)-\ln \left[G\left(t_{i} ; \underline{\eta}\right)\right]\right)\right]\right\} d \underline{\eta} d \theta,
\end{array}\right.\right.
\end{aligned}
$$

where $D(s)$ is defined in (47), $k^{-1}$ is defined in (24), $Q$ is defined in $(52), C(\theta ; \underline{\delta})$ and $D(\theta ; \underline{\delta})$ are defined in $(18), \pi(\underline{\eta} ; \underline{\gamma})$ is a subjective priors, and $G\left(y_{s} ; \underline{\eta}\right)$ and $A\left(t_{i} ; \theta, \underline{\eta}\right)$ are defined in (7) and (20), respectively, with $\underline{\eta}$ unknown.

It is immediate that $h^{*}\left(y_{s} \mid \underline{t}\right)$ cannot be expressed in closed form, and hence, it can be evaluated numerically.

The Bayesian prediction bounds for $Y_{s}$ are obtained by evaluating $P\left(Y_{s} \geq v \mid \underline{t}\right)$ for some positive value of $v$ as follows: $P\left(Y_{s} \geq v\right)=\int_{v}^{\infty} h^{*}\left(y_{s} \mid \underline{t}\right) d y_{s}$. Having obtained $P\left(Y_{s} \geq v \mid \underline{t}\right)$, a $100 \tau \%$ Bayesian predictive interval for $Y_{s}$ is then given by $P\left[L L \leq Y_{s} \leq U L\right]=\tau$, where $L L$ 
and $U L$ of the $100 \tau \%$ Bayesian predictive interval for $Y_{s}$ are obtained by solving the two equations

$P\left(Y_{s} \geq L L \mid \underline{t}\right)=\frac{1+\tau}{2} \quad$ and $\quad P\left(Y_{s} \geq U L \mid \underline{t}\right)=\frac{1-\tau}{2}$.

\section{Numerical Examples}

Consider $G(t ; \underline{\eta})=G(t ; \sigma)=1-\exp (-\sigma t)$, an exponential distribution with scale parameter $\sigma$. So that

$$
F(t ; \underline{\eta})=F(t ; \theta, \sigma)=[1-\exp (-\sigma t)]^{\theta}
$$

This implies to, $k=1, \underline{\eta}=(\sigma)$ and $\lambda(t ; \sigma)=\sigma$.

Estimation of the parameters and Bayes predictions of the lifetime lengths of all censored units in progressive sample from the EE could be computed as follows For given values of the parameters $\theta=2, \sigma=0.2, R(1)=0.96714$ and $h(1)=0.06138$ the sample size $n=30$, the observed sample size $m=24$ and censoring scheme $R_{i} ; i=1,2, \ldots, m$, a simulated progressive Type-II censored sample from the EE density given by (50), is generated according to the Balakrishnan and Sandhu (1995) algorithm. The sample and the corresponding censoring scheme were summarized in Table 1.

Table 1: Progressively Type-II Censored Sample

\begin{tabular}{|c|c|c||c|c|c|}
\hline$i$ & $t_{i}$ & $R_{i}$ & $i$ & $t_{i}$ & $R_{i}$ \\
\hline \hline 1 & 0.07453 & 2 & 13 & 5.29994 & 0 \\
\hline 2 & 1.31565 & 0 & 14 & 5.33113 & 0 \\
\hline
\end{tabular}




\begin{tabular}{|c|c|c||c|c|c|}
\hline 3 & 1.39383 & 0 & 15 & 5.70934 & 0 \\
\hline 4 & 1.53255 & 0 & 16 & 8.42614 & 0 \\
\hline 5 & 1.67901 & 0 & 17 & 9.04131 & 1 \\
\hline 6 & 2.11215 & 0 & 18 & 9.24132 & 0 \\
\hline 7 & 2.19984 & 0 & 19 & 9.54100 & 0 \\
\hline 8 & 2.38120 & 0 & 20 & 14.7919 & 0 \\
\hline 9 & 2.42607 & 1 & 21 & 19.0596 & 0 \\
\hline 10 & 2.65853 & 0 & 22 & 19.0932 & 0 \\
\hline 11 & 4.78904 & 2 & 23 & 23.1945 & 0 \\
\hline 12 & 5.01500 & 0 & 24 & 25.9794 & 0 \\
\hline
\end{tabular}

Two cases are considered based on the sample in Table 1, first is only exponent parameter $\theta$ is unknown and two parameters $\theta$ and $\sigma$ are unknown.

\subsection{Case (I): $\theta$ is unknown and $\underline{\eta}$ is known}

Assuming that, $\theta$ has a gamma prior $\frac{b^{a}}{\Gamma(a)} \theta^{a-1} e^{-b \theta}$, this implies to $C(\theta ; \underline{\delta})=\theta^{a-1}$ and $D(\theta ; \underline{\delta})=b \theta$.

Then, using the data in Table (1), the estimate of $\theta, R\left(t_{0}\right)$ and $h\left(t_{0}\right)$ for $t_{0}=1$ are computed from (9) and (10) with $a=7$ and $b=3.2$ and with three different LINEX constants as follows:

- $\quad$ ML estimates are $\hat{\theta}_{M L}=1.51738, \hat{R}_{M L}\left(t_{0}\right)=0.92508$ and $\hat{h}_{M L}\left(t_{0}\right)=0.11101$.

- $\quad$ Bayesian estimates under SE are $\hat{\theta}_{B S}=1.61756, \hat{R}_{B S}\left(t_{0}\right)=0.92994$ and $\hat{h}_{B S}\left(t_{0}\right)=0.10311$.

- Bayesian estimates under LINEX with constant $=2$ are

$$
\hat{\theta}_{B L_{1}}=1.54688, \hat{R}_{B L_{1}}\left(t_{0}\right)=0.92891 \text { and } \hat{h}_{B L_{1}}\left(t_{0}\right)=0.10206 \text {. }
$$

- Bayesian estimates under LINEX with constant $=0.001$ are

$$
\hat{\theta}_{B L_{2}}=1.61752, \hat{R}_{B L_{2}}\left(t_{0}\right)=0.92994 \text { and } \hat{h}_{B L_{2}}\left(t_{0}\right)=0.10311 \text {. }
$$

- Bayesian estimates under LINEX with constant $=-0.001$ are 


$$
\hat{\theta}_{B L_{3}}=1.61760, \hat{R}_{B L_{3}}\left(t_{0}\right)=0.92995 \text { and } \hat{h}_{B L_{3}}\left(t_{0}\right)=0.10311 \text {. }
$$

Also, the Bayesian predictions of the lifetime lengths of all censored units in progressive sample are computed according to (43) as shown in Table 2.

Table 2: Bayesian Prediction of Times to Failure of Censored Units Based on SE and LINEX loss functions for Case I

\begin{tabular}{|c|c|c|c|c|}
\hline \multirow{2}{*}{} & \multirow{2}{*}{ SE } & \multicolumn{3}{|c|}{ LINEX } \\
\cline { 3 - 5 } & & 2 & -0.001 & 0.001 \\
\hline \hline$U_{1: R_{1}}$ & 5.59673 & 2.20029 & 5.60246 & 5.59103 \\
\hline$U_{2: R_{1}}$ & 11.6641 & 4.04986 & 11.6826 & 11.6456 \\
\hline \hline$U_{1: R_{9}}$ & 8.68524 & 3.88560 & 8.69986 & 8.67038 \\
\hline \hline$U_{1: R_{11}}$ & 8.00331 & 5.85940 & 8.00752 & 7.99912 \\
\hline$U_{2: R_{11}}$ & 13.5540 & 7.22102 & 13.5713 & 13.5367 \\
\hline$U_{1: R_{17}}$ & 14.2952 & 10.2842 & 14.3080 & 14.2831 \\
\hline
\end{tabular}

\subsection{Case (II): $\theta$ and $\eta$ are unknown}

Suppose that $\theta$ and $\sigma$ are independent and both having gamma priors $\underline{\delta}=(7,3.2)$ and $\underline{\gamma}=(10,1.5)$, this implies to $C(\theta ; \underline{\delta})=\theta^{a-1}, D(\theta ; \underline{\delta})=b \theta$ and $\pi(\sigma, \underline{\gamma})=\frac{b^{a}}{\Gamma(a)} \theta^{a-1} e^{-b \theta}$

Then, using the data in Table (1), the estimate of $\theta, \sigma, R\left(t_{0}\right)$ and $h\left(t_{0}\right)$ for $t_{0}=1$ are computed from (15), (16) and (22) with three different LINEX constants as follows

- $\quad$ ML estimates are $\hat{\theta}_{M L}=1.09481, \hat{\sigma}_{M L}=0.12577, \hat{R}_{M L}\left(t_{0}\right)=0.90348$ and $\hat{h}_{M L}\left(t_{0}\right)=0.10976$.

- Bayesian estimates under SE are

$$
\hat{\theta}_{B S}=1.66132, \hat{\sigma}_{B S}=0.20906, \hat{R}_{B S}\left(t_{0}\right)=0.93013 \text { and } \hat{h}_{B S}\left(t_{0}\right)=0.10437 \text {. }
$$

- Bayesian estimates under LINEX loss function with constant=2 are

$$
\hat{\theta}_{B L_{1}}=1.5570, \hat{\sigma}_{B L_{1}}=0.20772, \hat{R}_{B L_{1}}\left(t_{0}\right)=0.92908 \text { and } \hat{h}_{B L_{1}}\left(t_{0}\right)=0.10327 \text {. }
$$

- Bayesian estimates under LINEX loss function with constant $=0.001$ are

$$
\hat{\theta}_{B L_{2}}=1.66126, \hat{\sigma}_{B L_{2}}=0.20906, \hat{R}_{B L_{2}}\left(t_{0}\right)=0.93013 \text { and } \hat{h}_{B L_{2}}\left(t_{0}\right)=0.10437 \text {. }
$$


- Bayesian estimates under LINEX loss function with constant $=-0.001$ are

$$
\hat{\theta}_{B L_{3}}=1.66138, \hat{\sigma}_{B L_{3}}=0.20906, \hat{R}_{B L_{3}}\left(t_{0}\right)=0.93013 \text { and } \hat{h}_{B L_{3}}\left(t_{0}\right)=0.10437 \text {. }
$$

Also, the Bayesian predictions of the lifetime lengths of all censored units in progressive sample are computed according to (37) shown in Table 3.

Table 3: Bayesian Prediction of Times to Failure of Censored Units Based on SE and LINEX loss functions for Case II

\begin{tabular}{|c|c|c|c|c|}
\hline & \multirow{2}{*}{ SE } & \multicolumn{3}{|c|}{ LINEX } \\
\cline { 3 - 5 } & & 2 & -0.001 & 0.001 \\
\hline$U_{1: R_{1}}$ & 3.82096 & 1.42312 & 3.82563 & 3.81625 \\
\hline$U_{2: R_{1}}$ & 9.33207 & 2.86466 & 9.35040 & 9.31384 \\
\hline \hline$U_{1: R_{9}}$ & 8.02786 & 3.74403 & 8.04242 & 8.01340 \\
\hline \hline$U_{1: R_{11}}$ & 7.50126 & 5.72874 & 7.50505 & 7.49749 \\
\hline$U_{2: R_{11}}$ & 12.6473 & 6.95432 & 12.6650 & 12.6296 \\
\hline$U_{1: R_{17}}$ & 14.1078 & 10.2434 & 14.1213 & 14.0944 \\
\hline
\end{tabular}

\section{References}

Abdel-Hamid, A. H., AL-Hussaini, E. K. (2009). Estimation in Step-Stress Accelerated Life Tests for the Exponentiated Exponential Distribution with Type-I Censoring. Computational Statistics and Data Analysis 53:1328-1338.

AL-Hussaini, E. K. (1999). Predicting Observables from a General Class of Distributions. Journal of Statistical Planning and Inference 79:79-91.

AL-Hussaini, E. K. and Al-Awadhi, F. (2010). Bayes Two-Sample Prediction of Generalized Order Statistics with Fixed and Random Sample Size. Journal of Statistical Computation and Simulation 80(1):13-28.

Ali Mousa, M. A. M., Jaheen, Z. F. (2002). Bayesian Prediction for Progressively Censored Data from the Burr Model. Statistical Papers 43:587-593.

Balakrishnan, N., Aggarwala, R. (2000). Progressive Censoring: Theory, Methods and Applications. Boston: Birkhauser. 
Balakrishnan, N., Sandhu, R. A., (1995). A Simple Simulation Algorithm for Generating Progressive Type-II Censored Samples. The American Statistician 49(2), 229-230.

Basak, I., Balakrishnan, N. (2009). Predictors of Failure Times of Censored Units in Progressively Censored Samples from Normal Distribution. Sankhya: The Indian Journal of Statistics 71-B:222-247.

Basak, I., Basak, P., Balakrishnan, N. (2006). On Some Predictors of Times to Failure of Censored Items in Progressively Censored Samples. Computational Statistics \& Data Analysis 50:1313-1337.

Gupta, R. C., Gupta, R. D., Gupta, P. L. (1998). Modeling Failure Time Data by Lehman Alternatives. Communications in Statistics-Theory and Methods 27(4):887-904.

Jaheen, Z. F. (2003). Prediction of Progressive Censored Data from the Gompertz Model. Communications in Statistics-Simulation and Computation 32(3):663-676.

Kakade, C. S., Shirke, D. T., Kundu, D. (2008). Inference for $\mathrm{P}(\mathrm{Y}<\mathrm{X})$ in Exponentiated Gumbel. Journal of Statistics and Applications 3(1-2):121-133.

Kim, C., Jung, J., Chung, Y. (2009). Bayesian Estimation for the Exponentiated Weibull Model under Type-II Progressive Censoring. Statistical Papers 52(1):53-70.

Madi, M. T., Raqab, M. Z. (2009). Bayesian Inference for the Generalized Exponential Distribution Based on Progressively Censored Data. Communications in Statistics-Theory and Methods. 38:2016-2029.

Persson, K., Ryden, J. (2010). Exponentiated Gumbel Distribution for Estimation of Return Levels of Significant Wave Height. Journal of Environmental Statistics 1:1-12.

Raqab, M. Z., Asgharzadeh, A., Valiollahi, R. (2010). Prediction for Pareto Distribution Based on Progressively Type-II Censored Samples. Computational Statistics and Data Analysis 54:1732-1743. 
Soliman, A. (2002). Reliability Estimation in a Generalized Life-Model with Application to the Burr-XII. IEEE Transactions on Reliability 51(3):337-343.

Wu, S-J., Chen, D-H, Chen, S-T (2006). Bayesian Inference for Rayleigh Distribution under Progressive Censored Sample. Applied Stochastic Models in Business and Industry 22:269-279. 